\title{
Second Law of Thermodynamics Formalism Applied to Finite Duration through Cycles of Living Dissipative Systems
}

\author{
Jorge Antonio Montemayor-Aldrete, Pablo Ugalde-Vélez², Marcelo Del Castillo-Mussot, \\ Gerardo Jorge Vázquez ${ }^{1}$, Ernesto Federico Montemayor-Varela ${ }^{3}$ \\ ${ }^{1}$ Departamento de Estado Sólido, Instituto de Física, Universidad Nacional Autónoma de México, México, D.F., \\ México \\ ${ }^{2}$ Departamento de Materiales (CBI), Universidad Autónoma Metropolitana Azcapotzalco, México, D.F., México \\ ${ }^{3}$ Instituto Nacional de Perinatología, México, D.F., México \\ Email: jorge@fisica.unam.mx
}

Received 19 September 2014; revised 20 October 2014; accepted 7 November 2014

Academic Editor: Krishna Midde, University of California San Diego, USA

Copyright (C) 2014 by authors and Scientific Research Publishing Inc.

This work is licensed under the Creative Commons Attribution International License (CC BY).

http://creativecommons.org/licenses/by/4.0/

(c) (i) Open Access

\begin{abstract}
A simple and general theory to describe basic irreversible thermodynamic aspects of aging in all dissipative living is presented. Any dissipative system during its operation continuously loses efficiency by the production of structural or functional defects because of the second law of thermodynamics. This continuous loss of efficiency occurs on all the dissipative systems through the realization of specific functional cycles, leading to a maximum action principle of any system involving the Planck's constant during their total dissipative operation. We applied our theory to the calculation of men and women lifespans from basal metabolic rate per unit weight and to the calculation of a new aging parameter per cycle of some human organs or physiological functions. All microscopic theory of the aging of living beings should be consistent with the second law of the thermodynamics. In other words, the operation of the biological self-organized structures only implies a delay in which the dissipative biological systems outside of equilibrium approach inexorably to the thermodynamic equilibrium obeying the second law of the thermodynamics.
\end{abstract}

\section{Keywords}

Aging, Functional Damage, Entropy Production Rate, Dissipative Structures, Dissipative Cycles 


\section{Introduction}

From Schrödinger's classic work published in 1944 [1] it became clear that life, and not only the not-living systems like cyclic thermal machines, obeys the first and second laws of thermodynamics. Relative to the human body he writes, "Energy is needed to replace not only the mechanical energy of our bodily exertions, but also the heat we continually give off to the environment. That our body gives off heat is not accidental, but essential. This is precisely the manner in which we dispose of the surplus entropy we continually produce in our physical life process, and seems to suggest that the higher temperature of the warm-blooded animal includes the advantage of enabling it to get rid of its entropy at a quicker rate so that it can afford a more intense life process". The basic same ideas were expressed in a different paragraph using similar expressions: "organization of living organisms is maintained by extracting 'order' from the environment” and he also asks: "How would we express in terms of the statistical theory the marvelous faculty of a living organism, by which it delays the decay into thermodynamical equilibrium (death)?" Finally, he introduced the idea of an "aperiodic crystal", and the microcode of which Schrödinger spoke have become the DNA and the genetic code of today. It is well known that Watson and Crick, co-discoverers of the structure of DNA, each respectively acknowledged the mentioned book as a source of inspiration for their initial researches.

The next step of the theoretical conception of living systems or non-living dissipative systems in a thermodynamical scheme was due to the Prigogine work in 1945, when the theorem of minimum entropy production applicable to non-equilibrium stationary states is published [2]. According to Prigogine this theorem gives a clear explanation of the analogy relating the stability of equilibrium thermodynamical states and the stability of biological systems, such as that expressed in the concept of "homeostasis" proposed by Claude Bernard. The notion of "dissipative structure” was elaborated by him in 1967 [3], and in 1971, he concluded: “The formation of dissipative coherent structures, for sufficient far-from-equilibrium conditions, appears as a quite unexpected manifestation of 'disorder to order' processes, far from equilibrium, but conforming to the second law of thermodynamics” [4]. In the words, Professor Stig Claesson [5] states that: "Prigogine has been particularly captivated by the problem of explaining how ordered structures-biological systems, for example-can develop from disorder... which display order in both time and space, and which are stable to perturbations. Prigogine has called these systems dissipative systems, because they are formed and maintained by the dissipative processes which take place because of the exchange of energy between the system and its environment, and because they disappear if that exchange ceases... The method which Prigogine has used to study the stability of the dissipative structures to perturbations is of very great general interest. It makes it possible to study the most varied problems, such as city traffic problems, the stability of insect communities, the development of ordered biological structures... to mention but a few examples".

Additionally, to the previous information, it is an almost universal experimental fact that stationary states corresponding to dissipative structures do not last forever, despite being stable against fluctuations. Gradually, stationary states start to monotonically decline and eventually stop working to reach the thermodynamic equilibrium. For non-living dissipative systems, these processes could be defined as a process of intrinsic progressive loss of functionality, which conducts to ceasing of working properly, and eventually to failure [6]. For the living dissipative systems, this process of intrinsic progressive loss of organic function is reflected at the population level as an increase in the likelihood of death and a decline in the production of offspring [7] [8].

Given the generality of the finite duration of stationary states in dissipative structures within irreversible thermodynamic processes, the following steps in a thermodynamical scheme, it is obligated. Let us try to use the second law of thermodynamics to describe the following facts. Section 2 contains a thermodynamical background necessary for our model. In Section 3, we present some empirical thermodynamical observations of living systems and present our very general theoretical formalism of an average dissipation rate theory taking into account the cyclic nature of living beings. In Section 4, we analyze in our formalism data comparing lifespans of human beings (females and males) and different human organs and physiological functions. Section 5 is devoted to conclusions.

\section{Thermodynamical Background}

First, let us make a brief synthesis of relevant general thermodynamical results.

The first law reads,

$$
\mathrm{d} U=T \mathrm{~d} S-p \mathrm{~d} V
$$


where the internal energy of the system is $U, T$ is temperature in Kelvin scale, $S$ the entropy, $p$ the pressure acting on the homogeneous system and $V$ the volume. The second law defines entropy as

$$
\mathrm{d} S=\frac{\mathrm{d} Q_{\mathrm{rev}}}{T}
$$

with $Q_{\text {rev }}$ as the reversible heat flow.

Also we know [9] [10] that the rate of entropy production denoted by $\frac{\mathrm{d}_{i} S}{\mathrm{~d} t}$, also can be expressed as a function of generalized fluxes $J_{i}$ and generalized forces $X_{i}$ :

$$
\dot{S}=\frac{\mathrm{d}_{i} S}{\mathrm{~d} t} \equiv \sum_{i} J_{i} X_{i}
$$

From this equation, it is possible to define the Raleigh's dissipation function [10], $\Phi_{R}$ as

$$
\Phi_{R} \equiv T \dot{S}_{i}
$$

This represents the heat generation inside a system, and could be experimentally measured. For biological dissipative structures far from thermodynamic equilibrium, it is convenient to write Equation (4) per unit volume or per unit weight;

$$
\varnothing_{R}=T \dot{s}_{i}
$$

Prigogine [9] [11], by starting from Equation (3), shows that

$$
\frac{\mathrm{d}_{x} P}{\mathrm{~d} t} \leq 0
$$

where $P \equiv \frac{\mathrm{d}_{i} S}{\mathrm{~d} t}$ and the symbolism given by $\frac{\mathrm{d}_{x} P}{\mathrm{~d} t}$ denotes the time variation in $P$ due to a variation on the forces. This result is valid provided the system is subject to time-independent boundary conditions, and it is the extension of the minimum entropy-production property to the non-linear domain of irreversible processes. However, in contrast to inequality for linear irreversible thermodynamics $\left(\frac{\mathrm{d} P}{\mathrm{~d} t} \leq 0\right)$ inequality Equation (6) does not imply the stability of the steady-state, primarily because in the general case $\mathrm{d}_{x} P$ is not the differential of a state function.

\section{Average Dissipation Rate Theory}

\subsection{General Empirical Facts}

It has been experimentally observed long time ago that during the time development of living systems, the dissipative Raleigh function per unit volume $\varnothing_{R}$ grows rapidly with the beginning (after birth) of the living system, then later it attains a maximum value, and after that it decreases until a steady-state value is reached. However, this steady-state does not last forever, and eventually the system fails or dies. See for instance Figure 1 for the human normal basal metabolic rate at different ages for each sex.

The general schematic behavior of self-organized living dissipative structures is shown in Figure 2, where the specific (per unit volume) production rate of entropy versus time is presented. In more detail, the curve shows four chronological stages: 1) An initial transient stage, where $\dot{s}$ grows in a more or less exponential way; after some time, the production rate of entropy reaches its maximum value and becomes decreasing. In this stage, the organism is getting bigger and more developed through time than before; 2 ) Then we have a stationary or steady-state stage, where $\dot{s}$ is approximately constant, which corresponds to a dynamical dissipative equilibrium state of the system. In this stage, the specimen already arrived to adulthood, and aging occurs in a slow rate; 3 ) "Aging in the mature stage" is characterized by an approximate linear decrease of $\dot{s}$ with time, corresponding to an average linear decreasing of physiological response of all the organs or subsystems of the living being; 4) Finally, it occurs a stage corresponding to catastrophic failure or destruction of the biological dissipative structure.

In our formalism, the initial time is the instant when the "egg" is formed, which depends on the type of living being. For instance, in a mammal, we consider that time starts with the fetus development, and for the birds, the 


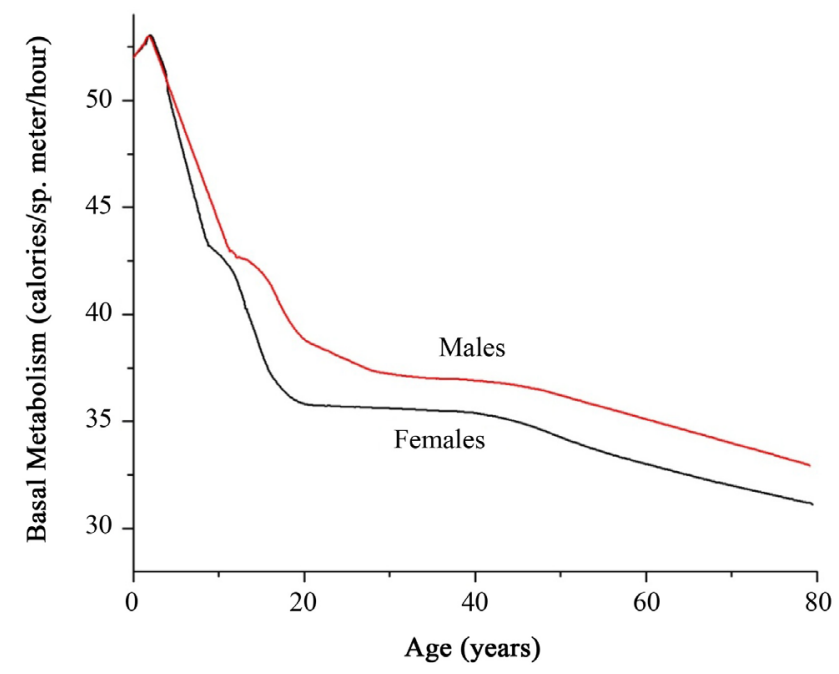

Figure 1. Normal basal metabolic rates at different ages for each sex [12].

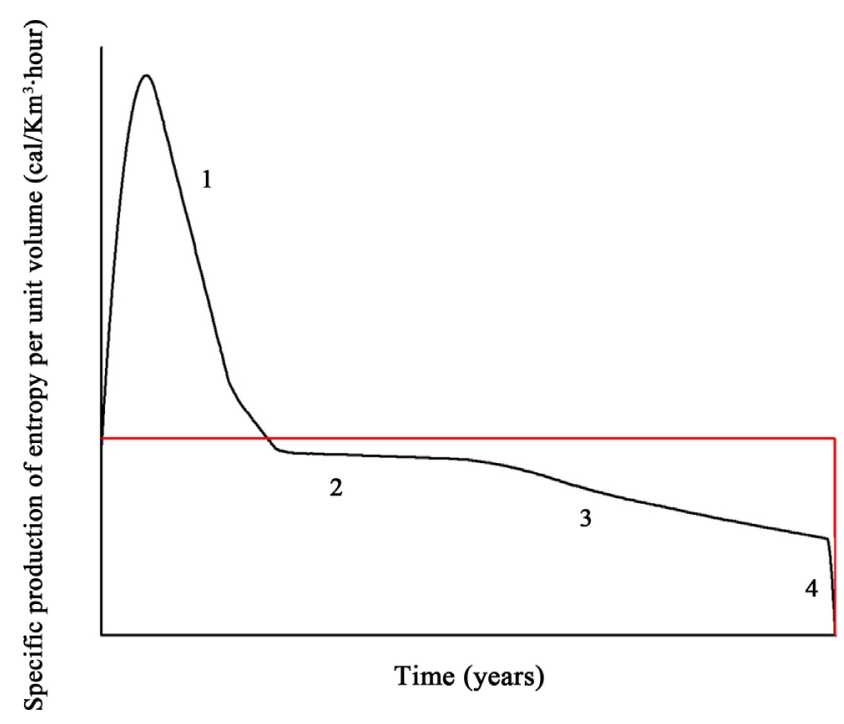

Figure 2. Schematic diagram of specific (per unit volume) production rate of entropy vs. time showing four chronological stages; 1 -initial growing, 2 - stationary or steady-state stage, (adulthood), 3-aging in the mature stage, and 4-catastrophic failure or destruction. The rectangle should have the same area to apply Calculus mean value theorem.

process depends on the heat received by the egg in the incubation process.

It is widely observed that most living dissipative systems perform functional cycles. For example in mammals breathing, heartbeat, digestive processes, etc. are cyclic processes. Of these, the most important functional cycle is the relative to the rate of arrival of nutrients and oxygen to each and every one of the cells that make up every living system. The heartbeats promote, throughout the body, a distribution of free energy per unit time which allows meeting the needs of the dissipative system. Clearly, for each process that occurs within the system, the length of each cycle varies with the age of each living being and that may depend on the specific activities carried out at all times. As a first approximation, the case of the cyclic dissipative systems will be analyzed, under the assumption that a large population of them can be represented by average values through their working life. Based on the second law of thermodynamics, is considered here that any living being gradually wears down and 
eventually stops working as it previously did (in a similar way that occurs for an internal combustion engine, which also operates through cycles).

During their operation dissipative systems perform internal processes of a cyclic nature that could be repeated hundreds, thousands or thousands of millions of times, etc. The dissipative structures go through a maximum number of cycles, $N_{\max }$ before a catastrophic failure occurs. Each type of dissipative system has its own characteristic maximum number of cycles. In other words, let us state this important general fact: Every dissipative structure working by functional cycling necessarily generate a net quantity of functional entropy or functional disorder in every cycle and, therefore, the structure cannot last longer than a maximum time of continuous work characteristic of each structure. In other words, the total quantity of energy dissipated as heat by any dissipative structure during its total working time has a maximum value. This finite time of continuous operation is due to the increase of functional entropy or functional disorder which eventually causes the breaking of the dissipative machine. We will show that any dissipative system realizing cycles obeys a maximum action principle, which value is characteristic of each system.

If we consider the whole process to take place at a constant temperature $T$, then from Figure 2, it is clear that for each particular dissipative system, the area under its curve is the maximum generated entropy. In the following it will be considered that when a species has reached its evolutionary stability, their adult members have common dissipative thermodynamic characteristics. Then, the statistical description of any of its living members (any age) can be obtained by using estimation or measuring the maximum total free energy dissipated through his life of the individuals who have been the longest-lived of the species.

In mathematical language

$$
\int_{t_{i}}^{t_{f}} \frac{\mathrm{d} s}{\mathrm{~d} t} \mathrm{~d} t=\frac{K_{s}}{T}
$$

where $K_{s}$ is the energy dissipated during the whole working process for each dissipative type of machine or living system, and $t_{i}$ and $t_{f}$ are the initial and final times, respectively. If we make use of the Calculus mean value theorem, then

$$
\frac{\overline{\mathrm{ds}}}{\mathrm{d} t}\left(t_{f}-t_{i}\right)=\frac{K_{s}}{T}
$$

where $\frac{\overline{\mathrm{d} s}}{\mathrm{~d} t}$ is the average value of the production rate of specific entropy for our system between times $t_{i}$ and $t_{f}$, where the graphic meaning of Equation (8) in the scheme of Figure 2 is obvious. By using Equation (5), for average values, into Equation (8); we have that

$$
\frac{\overline{\varnothing_{R}}}{T}\left(t_{f}-t_{i}\right)=\frac{K_{s}}{T}
$$

Thus, the total operation time of the dissipative system is given by

$$
\left(t_{f}-t_{i}\right)=\frac{K_{s}}{\bar{\varnothing}_{R}}
$$

Which shows in a direct way that a dissipative system has a maximum of continuous time operation inversely proportional to the specific dissipative Raleigh's function of the system, and directly proportional to their $K_{s}$, which is characteristic of each dissipative system.

\subsection{Cyclic Nature of Dissipative Systems}

It is clear that many dissipative structures realize functional cycles, which repeat over and over during its whole period of operation, before fail. Assuming, for simplicity, that the cycles are identical, then, from Equation (9):

$$
\frac{\overline{\varnothing_{R}}}{T} \tau\left[\frac{t_{f}-t_{i}}{\tau}\right]=\frac{K_{s}}{T}
$$

Here $\tau$ is the average time which characterizes the cycle duration. Equation (11) means that every time the 
living machine performs one of the $N_{\max } \equiv\left[\frac{t_{f}-t_{i}}{\tau}\right]$ cycles, it produces a quantity of specific entropy, $\frac{\overline{\varnothing_{R}}}{T} \tau$.

In the sake of mathematical simplicity, constant or average duration of the functional cycles through life will be assumed, even though we know that during adulthood metabolism decreases in time, which causes that the time duration of cycles also diminish.

The existence of an upper limit to the number of the operation cycles implies also the existence of accumulative damage and aging processes. This damage and aging processes cause the continuous decreasing on the power that the dissipative can handle through time and from empirical observation is clear that the aging rate is different and characteristic for each type of system.

For auto-organized systems, there are many phenomenological observations, which show that the macroscopic symptoms of aging begin to appear after the auto-organized dissipative structure attains adulthood [13]. This stage corresponds to the stage 2 of Figure 2 previously defined. In the stage 3, the specimen already arrived to adulthood, and the production rate of specific entropy will be a decreasing function of time.

Taking into account such empirical information from macroscopic signs of aging it is possible to make an approximation about the derivative in time $\dot{s}$, which can be expressed as a function relative to the steady-state rate of entropy production $\overline{(\dot{s})}$ like a bilinear function of $\alpha$ and $t$,

$$
\dot{s}=\dot{s}(\alpha, t)
$$

where $\alpha$ is an aging parameter measuring the functional decay of the dissipative system in each cycle. It is obvious that the simplest expression capable to deal with the decreasing on the rate of entropy production by aging in stage 3 is

$$
\dot{s}(t)=\overline{(\dot{s})}-\alpha * \overline{(\dot{s})}\left[\frac{t-t_{s d}}{\tau}\right]
$$

Here $t_{s d}$ indicates the time where steady-state begins to decay and $t_{s d}$ corresponds to the beginning of stage 3. Equation (13) becomes:

$$
\dot{s}(t)=\overline{(\dot{s})}\left[1-\alpha\left(\frac{t-t_{s d}}{\tau}\right)\right]
$$

which clearly states that every dissipative structure necessarily suffers accumulative damage during its operation. We can say that the dissipative system has some functional memory, in the sense that cannot be rejuvenated. That is, $\dot{s}(t)$ decreases with the accumulated time of operation of the dissipative system at a rate given by $\alpha$.

Neglecting the contribution of the collapse stage 4 (assuming that this last stage is extremely short as compared with the stage 3), also from Equation (14) it is easy to define a new physical macroscopically variable related to the steady-state of entropy production; the fractional remaining functionality:

$$
\Pi_{r e}(t) \equiv \frac{\dot{s}(t)}{\overline{(\dot{s})}}
$$

Therefore,

$$
\Pi_{r e}(t)=1-\alpha *\left(\frac{t-t_{s d}}{\tau}\right)
$$

From this equation, it is clear that when the dissipative system collapses, $\Pi_{r e}\left(t_{f}\right)=0$. This result implies that $\alpha$ can be evaluated, for instance, from the total failure condition as follows:

$$
\alpha=\frac{\tau}{\left(t_{f}-t_{s d}\right)}
$$

By using Equation (17) into Equation (14)

$$
\dot{s}(t)=\overline{(\dot{s})}\left[1-\left(\frac{t-t_{s d}}{t_{f}-t_{s d}}\right)\right]
$$


which describes the time evolution of $\dot{s}(t)$ after steady-state ceases $\left(t>t_{s d}\right)$. Also, it is possible to define a functional damage, $D(t)$, as

$$
D(t) \equiv\left(\frac{t-t_{s d}}{t_{f}-t_{s d}}\right)
$$

which could be expressed as a function of the total cycle number at failure condition $N_{T}$ characterizing the duration of each structure:

$$
D\left(t_{n}\right)=\frac{n}{N_{T}}=\frac{W_{n}}{W_{N_{T}}}
$$

Here $w$ is the work per cycle, $W_{N_{T}} \equiv N_{T} w$, and $W_{n} \equiv n w$; with $N_{T}$ as the total cycle number at failure condition. By using Equations (17)-(19) in Equation (16) we get

$$
\Pi_{r e}(t)+D(t)=1 \text {. }
$$

This equation shows, as expected, that fractional remaining functionality has high values, when the functional damage is low. For animals, these equations allow to see that at the time $t,\left(t>t_{s d}\right)$, the specific power capable to be used or assimilated by the dissipative system, $\Pi_{r e} * \overline{(\dot{s})} T$, is lower than $\overline{(\dot{s})} T$; because of the thermodynamical limitations imposed by the functional damage $D(t)$ produced by the operation of the dissipative system itself. It is clear, that $D(t) * \overline{(\dot{s})} T$ is the specific power that the dissipative system is incapable to use or assimilate, after steady-state, because of the accumulated functional damage at timet.

\subsection{Maximum Action Principle}

Here, by using our formalism, it is possible to obtain an equation for the maximum entropy changes in a dissipative system as multiples of the Boltzmann's constant. For this purpose, let us employ another physical meaning of Equation (9) if we use Equation (5), together with $\dot{s}=\frac{\mathrm{d} Q_{i}}{\mathrm{~d} t}$ as the specific entropy production rate for the $i$-dissipative system, it is possible to obtain the following equation:

$$
\dot{\theta}_{i} t_{f i}=K_{s i}
$$

where $\dot{\theta}_{i} \equiv \frac{1}{M}\left(\frac{\mathrm{d} Q_{i}}{\mathrm{~d} t}\right), \quad Q_{i}$ is the dissipated heat, $M$ is the mass of the $i$-system and $t_{f i}$ is the failure time of the $i$-system. Therefore, Equation (22) can be written as

$$
\mathrm{d} Q_{i} t_{f i}=M K_{s i} \mathrm{~d} t \equiv h_{i \max }
$$

where $M K_{s i} \mathrm{~d} t \equiv h_{i \max }$ is a constant for each $i$-dissipative system and its physical meaning is the maximum action developed by the dissipative $i$-system during their total dissipative operation. Recall that the action concept which has dimensions of [ENERGY multiplied by TIME], comes from mechanics in relation to Integral Variational Principles of Least Action, which give place to the motion equations for any mechanical system [14].

Therefore, the total entropy generated during the continuous dissipative operation of the dissipative system, $\Delta S_{i}$, as a function of the maximum action, is

$$
\Delta S_{i}=\frac{1}{T}\left(\frac{h_{i \max }}{\mathrm{d} t}\right)
$$

Notice that physically and mathematically Equation (23) resembles the uncertainty principle due to Heisenberg;

$$
\Delta E \Delta t \geq \frac{h}{2 \pi}
$$

where $E$ is for the energy and $t$ for time, and $h$ is the Planck's constant.

By using Equation (25), into Equation (24) it is possible to obtain 


$$
\Delta S_{i}=k\left(\frac{h_{i \max }}{h / 2 \pi}\right)
$$

where $k$ is the Boltzmann's constant. Therefore, the total entropy generated during the continuous dissipative operation of the dissipative system, $\Delta S_{i}$, is a function of the ratio between the maximum action characteristic of each type of dissipative system, and the Planck's constant which is the minimum quantum action [14], times the Boltzmann's constant.

In the following paragraphs, a comparison between the theoretical results here obtained with experimental data, and other theoretical considerations will be made.

\section{Comparison between Experimental Data and Theory}

\subsection{Average Lifespan of Human Females and Males}

First, we will give a quantitative thermodynamic explanation to experimental facts that on average (for all current country populations) women live several years more than men. Later, we will discuss some thermodynamic implications of aging of humans and their internal organs. On one hand, from Figure 3, [15] it is immediate that the average basal metabolic rate in man throughout his existence is $40 \quad(\mathrm{Kcal} / \mathrm{sq} \cdot \mathrm{m} / \mathrm{h})$, and for women is 36.8 $(\mathrm{Kcal} / \mathrm{sq} \cdot \mathrm{m} / \mathrm{h})$. According to [16] the mean body area for European man and female are $1.9 \mathrm{~m}^{2}$ and $1.6 \mathrm{~m}^{2} \mathrm{re}-$ spectively. Also from reference [17] the average European male weight is $78.9 \mathrm{Kg}$ and for female the value is 65.8. Therefore, the average value for the basal metabolic rate per unit weight (bmr) for European man and women are $0.963(\mathrm{Kcal} / \mathrm{Kg} / \mathrm{h})$ and $0.9161(\mathrm{Kcal} / \mathrm{Kg} / \mathrm{h})$ respectively. Using Equation (10) with the consideration that for this case $K_{s}$ (which is the energy dissipated during the whole working process for each dissipative type of living system has the same value for the human species), we have the following theoretical expression for the ratio between the span of life of European human females $t_{\mathrm{SLFem}}$ and the span of life of European human males $t_{\mathrm{SL} \text { Male }}$ :

$$
\left(\frac{t_{\mathrm{SL} \mathrm{Fem}}}{t_{\mathrm{SL} \text { Male }}}\right)=\frac{0.963}{0.9161}=1.051,
$$

On the other hand, taking into account the female and male life expectancy of all the members of the European Union (EU) [18] and their respective population, we obtain the average ratio for the female and male life

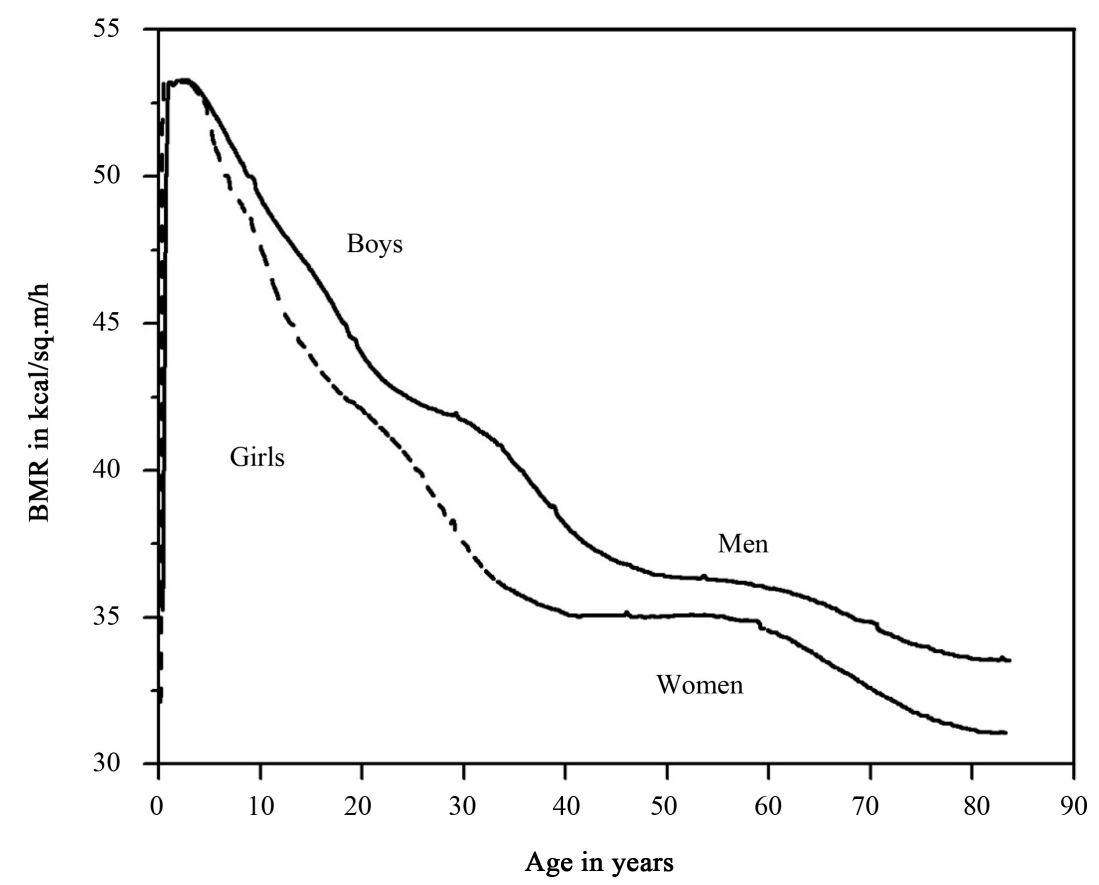


expectancy for the EU,

$$
\left(\overline{\frac{\tau_{\mathrm{LE} \mathrm{Fem}}}{\tau_{\mathrm{LE} \mathrm{Male}}}}\right)=1.077
$$

Therefore, the percentage difference between the theoretical and the experimental data is $2.5 \%$, which is an excellent result.

There are two reasons for employing EU data; their data availability and very few premature deaths of that relatively rich, healthy and homogeneous population as compared to other regions of the world population. Premature deaths are due to crimes, war, disease, and malnutrition. For instance, in Sub-Saharan Africa, one of the worse health problems (if not the worst) is underweight infancy.

\subsection{Aging Rates of Human Organs}

Relative to the thermodynamic implications of aging of humans and their internal organs, in Figure 4, experimental data for Average Percent Physiological Function Remaining of different human organs and physiological functions, (as given by Cutler [13]) are shown as a percentage of maximum lifespan potential; In principle, all these organs obey the Equation (16), but since they form part of the whole system, in reality, they are interdependent.

From data of Cutler et al. [13] as shown in Figure 4, by using Equation (16) we can calculate the values for the aging parameter, $\alpha$ (for each organ). If we use the same failing time and cardiac cycle $\tau$ for each organ, then the ratios of different $\alpha$ do not depend on this choice. We choose $\tau=1 \mathrm{~s}$ and failing time $=100$ years, the results are shown in Table 1.

The data on Table 1 allow to describe the time dependence of the percentage functional remaining, $\pi_{r e}$, corresponding to different organs, from aging human bodies, is in full agreement with experimental data. According to the theory of evolution of species in a species that has reached stability, all internal organs (in general) are aging at the same rate. Therefore all deviation from that behavior obeys environmental, behavioral or dietary factors outside the prevailing natural diet and behavior in the environment where the species evolved. One possible factor for the variations on slopes in $\pi_{r e}$ function for different human organs with living time could depend to social conducts like smoking, taking of alcohol, overweight, etc., that causes deleterious effects on their operation. A physical interpretation of such results is as follows: The rate of aging of the nerves is 3.43 times smaller than the average rate of aging social vital lung capacity, or about 2.64 times lower than the social average rate of aging for the cardiac impulse.

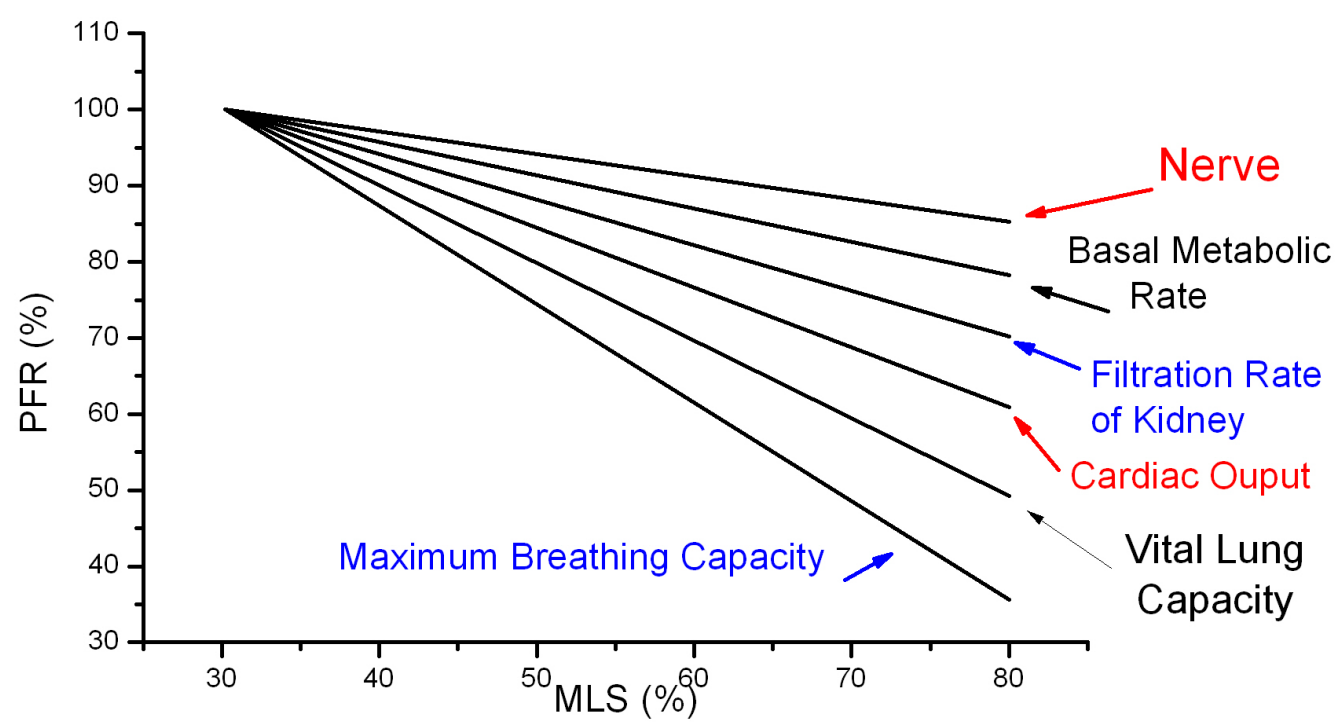

Figure 4. Experimental data for the average percentage remaining functionality (PFR) for different human organs and functions as a function of the percentage of maximum lifespan potential (as given by Cutler [13]). We point out that these straight lines also appeared in the original work. 
Table 1. Aging parameters, $\alpha$, which characterize the functional decay of the dissipative system in each cycle corresponding to different organs in human bodies.

\begin{tabular}{cc}
\hline Organ or Human Function & Values of $\alpha$ \\
\hline Nerve & $\alpha=9.38 \times 10^{-11}$ \\
Basal Metabolic Rate & $\alpha=1.388 \times 10^{-10}$ \\
Filtration Rate of Kidney & $\alpha=1.898 \times 10^{-10}$ \\
Cardiac Output & $\alpha=2.482 \times 10^{-9}$ \\
Vital Lung Capacity & $\alpha=3.22 \times 10^{-10}$ \\
Maximum Breathing Capacity & $\alpha=4.1 \times 10^{-10}$ \\
\hline
\end{tabular}

\section{Conclusions}

We identified general four chronological stages of self-organized living dissipative structures of the specific (per unit volume) production rate of entropy: 1) an initial more or less exponential transient stage, 2) stationary or steady-state stage, (adulthood), with slow aging, 3) aging in the mature stage” with an approximate linear decrease of $\dot{s}$ with time, and 4) catastrophic failure or destruction stage.

Based on the proposal of an average dissipation rate model for non-linear irreversible thermodynamics for dissipative structures, which goes through functional cycles, we conclude that the systems necessarily generate a maximum quantity of entropy (characteristic of each type) and, therefore, last a maximum quantity of time $t_{\mathrm{fi}}$. Such analysis allowed us to develop a new theory to describing the essential aspects of aging processes for any kind of dissipative systems.

The second law of thermodynamics has been used to explain the finite time duration of stationary states and simultaneously, both the processes of intrinsic progressive loss of functionality for non-living dissipative systems, and the processes of intrinsic progressive loss of organic function in the living dissipative systems. Some of the theoretical results (as Equation (10)) show in a direct way that all kinds of dissipative system have a maximum of continuous time operation inversely proportional to the specific dissipative Raleigh's function of the system, and directly proportional to their $K_{s}$, characteristic of each specific type of dissipative system. This equation allows us to show in a direct and quantitative way why on average the human female last longer than the human males.

We hope that future developments of simple concepts developed here, such as fractional remaining functionality (Equation (16)), could to make a contribution to develop programs for preventive health care. Different values for $\alpha$ corresponds to distinct aging rates on a given subsystem of human bodies. Then for a given organ (fundamental or not) it is possible to collect data of a human population and select the people with lower values for $\alpha$ than the population average, in order to make studies about their living and eating habits in order to develop preventive measurements for the rest of the population. It is clear that the previous analysis, for average population basal metabolism, in other words is a statistical scheme and it is not possible to distinguish personal behavior as for example: smoking, drinking or homosexual personal conducts.

Figures of the type of Figure 4 and the use of Equation (16), in principle allow to compare the general state of health of a concrete human being of $x$ age with regard to the general state of health at the same age corresponding with an average of humans being that later have arrived to the 100-year-old age. And also to determine the maximum duration of the person's life that moves away from the values that correspond to the possibility of arriving to a 100-year-old age. In short, for example, after evaluating the alpha corresponding to the centennials and knowing the average of the numerical values for the heart functionality in a 100-year-old population we can determine the value that would throw the use of Equation (16) for a specific person of $x$ age. It would allow it to evaluate the difference among the heart functionality from the centennials to that age and the subject in question. If we consider that the value of the heart functionality of the centennials is a limit value; then the use of Equation (16) for a specific person will determine us statistically their maximum duration of life. This analysis type can be a complementary useful tool, cheap and effective that cooperates to the standard evaluation procedures of the future health of big groups of human beings. For the time evolution of the basal metabolism on children is necessary a further independent study. 
Analogous to the quantum Heinsenberg's principle, we presented an empirical principle for maximum action of any dissipative system involving the Planck's constant.

We made use of average values of the basal metabolic rate per unit weight of European man and women to conclude that, on average, the human female last longer than the human males, with only a $2.5 \%$ of relative error. Also, we calculated the aging parameter measuring the functional decay of the dissipative system in each cycle from specific production of entropy of some human organs or physiological functions.

We assumed constant duration of the cycles in adulthood, even though we know that during adulthood, metabolism decreases in time. Then it will be desirable to refine our formalism to include different values of the average and the variance of the duration of the cycles in different stages of the living structures.

The substitution of organs as a way to increase the span of human life, we would have as limit the time implied by the aging rhythm of the original brain. In the same way that the inclusion of new pieces in an old car does not increase to infinite the lifespan of the car, since during the replacement process, a stage will be attained were the car rejuvenated no longer corresponds with the original car.

All microscopic theory of the aging of living beings should be consistent with the second law of the thermodynamics. In other words, the operation of the biological self-organized structures, is not more than an efficient mechanism of degradation of the free energy that we receive from the sun, and it only implies a delay in which the dissipative biological systems outside of equilibrium approach inexorably to the thermodynamic equilibrium obeying the second law of the thermodynamics.

\section{Acknowledgements}

JAMA specially wants to thanks to Prof. M. López de Haro for many years of deep discussions and arguments and by his contribution to final shaping of the ideas here developed.

\section{References}

[1] Schrödinger, E. (1992) What Is Life? With Mind and Matter and Autobiographical Sketches. Cambridge University Press, Cambridge. http://dx.doi.org/10.1017/CBO9781139644129

[2] Prigogine, I. (1945) Etude thermodynamique des phénomènes irréversibles. Thèse d'agrégation présentée en 1945 à l’Université Libre de Bruxelles. Desoer, Liège, 1947. Académie Royale de Belgique. Bulletin de la Classe des Sciences, 31, 600.

[3] Prigogine, I. (1969) Structure, Dissipation and Life. Theoretical Physics and Biology, Versailles, 1967. North-Holland Publ. Company, Amsterdam.

[4] Glansdorff, P. and Prigogine I. (1971) Structure, Stabilitéet Fluctuations, Masson, Paris. Thermodynamic Theory of Structure Stability and Fluctuations. Wiley and Sons, London.

[5] The Nobel Prize in Chemistry (1977) Ilya Prigogine. Award Ceremony Speech. Presentation Speech by Professor Stig Claesson of the Royal Academy of Sciences. (Translation from the Swedish Text)

[6] Feinberg, A.A. and Widom, A. (2000) On Thermodynamic Reliability Engineering. IEEE Transactions on Reliability, 49, 136-146.

[7] Kirkwood, T.B. and Austad, S.N. (2000) Why Do We Age? Nature, 408, 233-238. http://dx.doi.org/10.1038/35041682

[8] Partridge, L. and Gems, D. (2002) Ageing: A Lethal Side-Effect. Nature, 418, 921-921. http://dx.doi.org/10.1038/418921a

[9] Prigogine, I. (1967) Thermodynamics of Irreversible Process. Charles C. Thomas Publisher, Springfield.

[10] Glaser, R. (1999) Biophysics. Springer-Verlag, New York.

[11] Glansdorf, P. and Prigogine, I. (1971) Thermodynamical Theory of Structures, Stability and Fluctuations. Wiley Interscience, New York.

[12] Guytton, A.C. (1976) Textbook of Medical Physiology. 5th Edition, W. B. Saunders Company, Philadelphia-LondonToronto, 952.

[13] Cutler, R.G. (1978) Evolutionary Biology of Senescence. In: Behnke, J.A., Finch, C.E. and Moment, G.B., Eds., The Biology of Aging, Plenum Press, New York, 311-360. http://dx.doi.org/10.1007/978-1-4613-3994-6_20

[14] Sommerfeld, A. (1972) “Mechanics” Lectures on Theoretical Physics, Volume I. Paperback Edition, 6th Printing, Academic Press, Inc., Waltham, 181.

[15] Durnin, J.V.G.A. (1981) Basal Metabolic Rate in Man. University of Glasgow Glasgow Scotland. Joint FAO/WHO/ 
UNU Expert Consultation on Energy and Protein Requirements Rome, 5-17 October. http://www.fao.org/docrep/meeting/004/m2845e/m2845e00.htm

[16] Sparreboom, A. and Verweij, J. (2003) Paclitaxel Pharmacokinetics, Threshold Models, and Dosing Strategies. Journal of Clinical Oncology, 21, 2803-2804. http://dx.doi.org/10.1200/JCO.2003.99.038

[17] (2006) Health and Food. Fieldwork November, December 2005. Special Eurobarometer. European Commission. http://ec.europa.eu/public_opinion/archives/ebs/ebs_246_en.pdf

[18] WHO (2013) WHO Life Expectancy. http://apps.who.int/gho/data/node.main.688?lang=en 
Scientific Research Publishing (SCIRP) is one of the largest Open Access journal publishers. It is currently publishing more than 200 open access, online, peer-reviewed journals covering a wide range of academic disciplines. SCIRP serves the worldwide academic communities and contributes to the progress and application of science with its publication.

Other selected journals from SCIRP are listed as below. Submit your manuscript to us via either submit@scirp.org or Online Submission Portal.
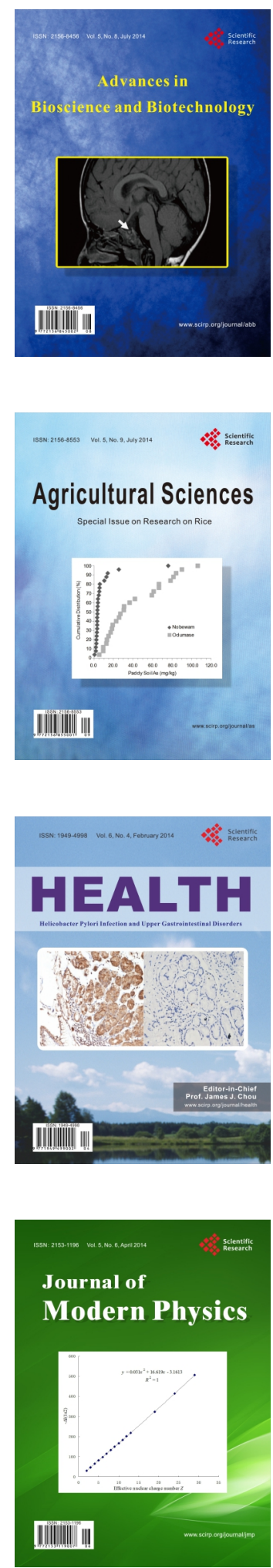
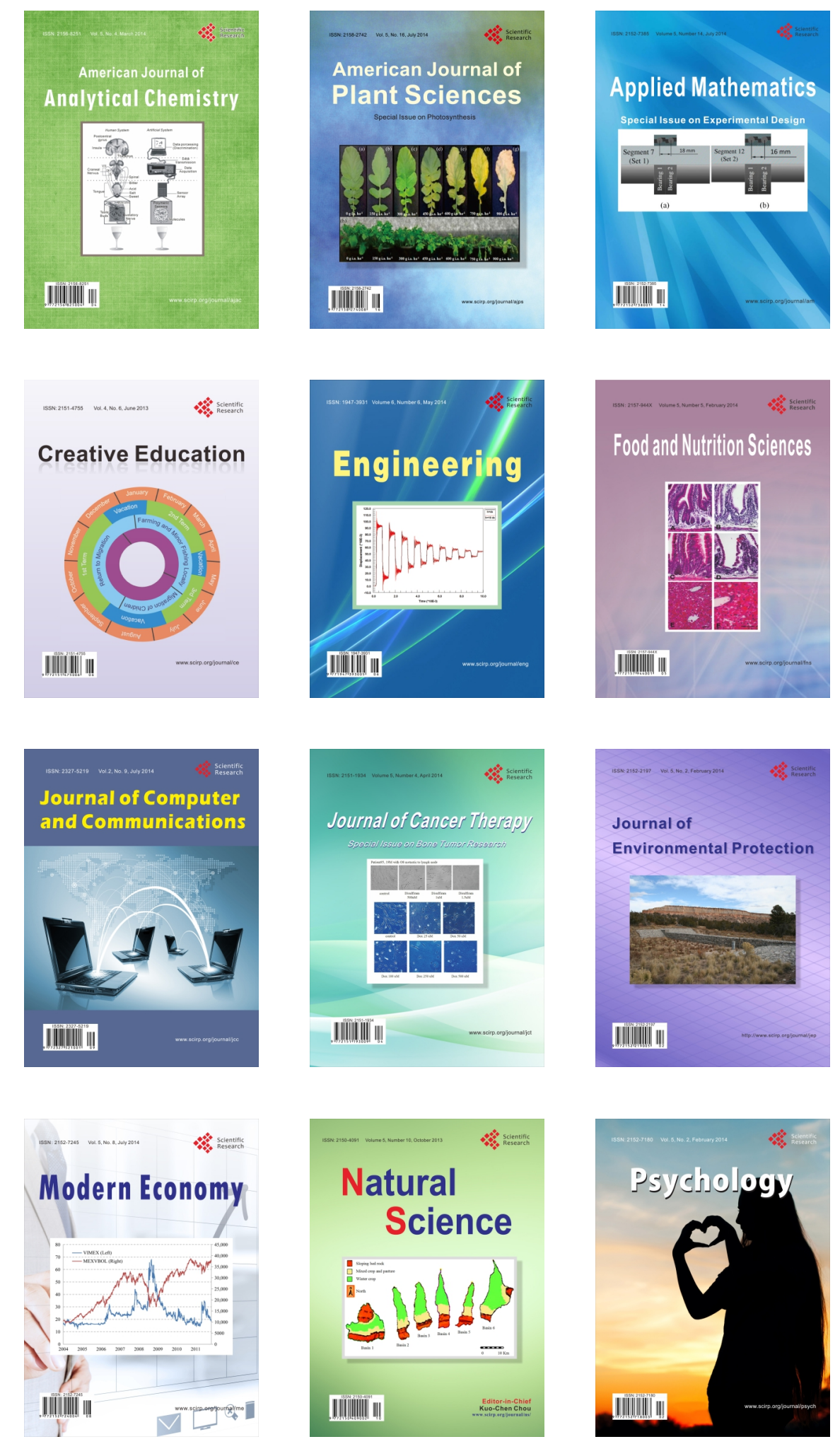